\title{
A IMPORTÂNCIA DA COMUNICAÇÃO DO RISCO DE DESASTRES NATURAIS: UM OLHAR SOBRE A REALIDADE MOÇAMBICANA
}

\author{
Manuel Pastor Francisco Conjo ${ }^{1}$ \\ David Benjamim Chichango ${ }^{2}$ \\ Paulo de Paula e Souza ${ }^{3}$ \\ Hortência Luísa Tole Tambo Foquiço ${ }^{4}$ \\ Isaura Viriato Samuel Matlava ${ }^{5}$ \\ Octávio Manuel de Jesus ${ }^{6}$
}

RESUMO: Considerando as situações vivenciadas face aos desastres naturais e a insegurança gerada pelos factores de risco em Moçambique, este artigo busca responder a questão: Qual é a importância da comunicação de risco na gestão dos desastres naturais em Moçambique? Discute-se o risco de desastres naturais como uma das principais preocupações das instituições ligadas à gestão de desastres naturais, a gestão de risco e a relação com a comunicação para a compreensão da comunicação de risco. Diante disso, objectivo geral é analisar como as práticas da comunicação de risco de desastres naturais se revelam no contexto moçambicano, incluindo a construção, implementação e importância de sistemas de informação e comunicação sobre o risco de desastres naturais em Moçambique. Para a materialização dos objectivos optou-se pela abordagem qualitativa aplicando o

\footnotetext{
${ }^{\text {I }}$ Doutorando em Ciência Florestal pela Universidade Federal de Viçosa - Minas Gerais -Instituição: Universidade Pedagógica de Maputo/Universidade Federal de Viçosa. Mestrado em Gestão Ambiental pela Universidade Pedagógica de Maputo - Moçambique. Bacharel e Licenciado em Ensino de Geografia pela Universidade Pedagógica de Maputo - Moçambique Técnico Superior em Higiene e Segurança no Trabalho e Meio Ambiente pela Ensine Moçambique. E-mail: pastorconjooo7@gmail.com

2 Mestrando em Gestão Ambiental, Faculdade de Ciências da Terra e Ambiente, Universidade Pedagógica de Maputo, Moçambique. Prof. de Geografia, Licenciado em Ensino de Geografia, Faculdade de Ciências da Terra e Ambiente, Universidade Pedagógica de Maputo, Moçambique. Email: davidchitlhango@gmail.com

3 Mestrando em Gestão Ambiental, Faculdade de Ciências da Terra e Ambiente, Universidade Pedagógica de Maputo, Moçambique. Gestor. Licenciado em Gestão e Direito Privado. Unirsity of South Africa. Pos- Graduação em Administração de Empresas, University of South Africa. E-mail: ppsouza@hotmail.com.

${ }^{4}$ Licenciada em Gestão Ambiental, Planificação e Desenvolvimento Comunitário pela Universidade Pedagógica, Delegação da Beira - Unilicungo. Consultora Ambiental.E-mail: hortambo@gmail.com 5 Mestranda em Gestão Ambiental, Faculdade de Ciências da Terra e Ambiente, Universidade Pedagógica de Maputo, Moçambique. Licenciada em Ciências Geológicas, Universidade Eduardo Mondlane, Maputo, Moçambique. Email: isauraviriatos@gmail.com.

${ }^{6}$ Doutor em Ciências Pedagógicas, moçambicano, funcionário do Ministério da Ciência, Tecnologia e Ensino Superior. Membro de Júri, Supervisor e Arguente nas Defesas de Monografias, Dissertações em Havana-Cuba e Maputo- Moçambique. Supervisor das teses de Doutoramento em Moçambique. Docente na Universidade Pedagógica de Maputo (desde 2006), categoria Professor Auxiliar. E-mail: Octaviomzoor@yahoo.es
} 
método de revisão bibliográfica da literatura científica sobre riscos, vulnerabilidade, desastres naturais, comunicação do risco incluindo pesquisa em documentos que tratam de matéria ligada a gestão de desastres naturais na República de Moçambique. Realizado o trabalho, os resultados mostram que a comunicação do risco de desastres naturais pode ser efectuada de forma escrita (jornais, cartas, relatórios, panfletos, etc.); verbal (conversas, apresentações, debates, etc.) ou não-verbal/ visual (filmes, gráficos, linguagem gestual, expressões faciais, entre outras). Os canais de comunicação ou são directos (face-to-face) ou indirectos (mediados) e actualmente são aplicados na comunicação do risco de desastres os Sistemas de Informação Geográfica. Moçambique possui um sistema de comunicação de riscos de desastres naturais dirigido pelo Instituto Nacional de Gestão do Risco de Desastres (INGD), que funciona em conexão com o Instituto Nacional de Meteorologia, com as comunidades locais e com a comunicação social. A comunicação do risco de desastres naturais assume hoje importante papel na gestão dos desastres naturais com várias finalidades e funções ao longo do ciclo do desastre, designadamente a prevenção/ preparação, aviso, intervenção e recuperação e, idealmente, deve abranger todos os processos de gestão do risco, desde a formulação do conhecimento, à decisão e implementação de acções.

Palavras-chave: Comunicação. Risco. Desastres naturais. Moçambique.

ABSTRACT: Considering the situations experienced in the face of natural disasters and the insecurity generated by risk factors in Mozambique, this article seeks to answer the question: What is the importance of risk communication in the management of natural disasters in Mozambique? The risk of natural disasters is discussed as one of the main concerns of institutions linked to natural disaster management, risk management and the relationship with communication for the understanding of risk communication. Therefore, the general objective is to analyze how natural disaster risk communication practices reveal themselves in the Mozambican context, including the construction, implementation and importance of information and communication systems on natural disaster risk in Mozambique. To materialize the objectives, a qualitative approach was chosen, applying the literature review method of the scientific literature on risks, vulnerability, natural disasters, risk communication, including research in documents dealing with matters related to natural disaster management in the Republic of Mozambique. Once the work has been carried out, the results show that communication of the risk of natural disasters can be done in writing (newspapers, letters, reports, pamphlets, etc.); verbal (conversations, presentations, debates, etc.) or non-verbal/visual (films, graphics, sign language, facial expressions, among others). Communication channels are either direct (face-to-face) or indirect (mediated) and Geographic Information Systems are currently used in the communication of disaster risk. Mozambique has a natural disaster risk communication system run by the National Institute of Risk of Disaster Management (INGD), which works in conjunction with the National Institute of Meteorology, local communities, and the media. The communication of natural disaster risk today assumes an important role in the management of natural disasters with various purposes and functions throughout the disaster cycle, 
including prevention/preparation, warning, intervention, and recovery and, ideally, should cover all management processes from the formulation of knowledge to the decision and implementation of actions.

Keywords: Communication. Risk. Natural disasters. Mozambique.

\section{INTRODUÇÃO}

O Quadro de Acção de Sendai para a Redução de Riscos de Desastres 2015-2030, assinado durante a Terceira Conferência da ONU para a Redução de Riscos de Desastres, realizada na Japão, em Março de 2015, apresenta compromissos e metas, sendo que as quatro prioridades de acção previstas nesse novo documento são, em síntese, compreender os riscos de desastres; fortalecer a governança de risco de desastres; investir na redução de risco de desastres para a resiliência; e melhorar a preparação aos desastres para uma resposta mais eficaz.

A comunicação de riscos de desastres encontra-se inserida nessas prioridades de acção e está presente, em diversas passagens do documento. Por meio da comunicação de riscos será possível garantir o acesso à informação sobre os riscos, a transferência de conhecimentos acerca desses riscos e a concepção de um modelo de educação, capaz de tornar as comunidades mais resilientes aos desastres.

Segundo SANDMAN (1986) apud SOUSA (2013), a comunicação do risco, além de actuar na redução dos riscos e dos danos, desempenha ainda um papel relevante na consciencialização, tomada de decisão e posicionamento dos diferentes intervenientes, em relação aos riscos.

Neste contexto, a comunicação do risco tem-se afirmado como uma área de grande relevância no quotidiano das sociedades actuais, desempenhando um papel central na forma de análise, compreensão, tomada de decisão, implementação e regulação das intervenções sobre o risco de desastres (SOUSA 2013:2).

De acordo com SOBRAL et al (2010), desde o fim da Segunda Guerra Mundial cerca de 1.200 desastres naturais afectaram a vida de mais de 2,3 milhões de pessoas em todo o mundo, causando muitos danos humanos e severas perdas econômicas.

Considerando as situações vivenciadas face aos desastres naturais e a insegurança gerada pelos factores de risco em Moçambique, este artigo busca responder a questão: 
Qual é a importância da comunicação do risco de desastres naturais em Moçambique? Discute-se o risco de desastres naturais como uma das principais preocupações das instituições ligadas a gestão de desastres naturais, a gestão de risco e a relação com comunicação, para a compreensão da comunicação de risco. Diante disso, tem-se como objectivo analisar como as práticas da comunicação de risco se revelam no contexto moçambicano.

\section{RISCO, DESASTRES NATURAIS E VULNERABILIDADE}

\subsection{Interpretações acerca do risco}

BERNSTEIN (1996), apud SOUSA (2013), sugere que a origem etimológica do termo risco deriva do italiano risicare, que significa ousar ou desafiar, no entanto, para este autor, não existe um consenso sobre o tema, entre a comunidade científica.

CUNHA (2002), relaciona o aparecimento e disseminação deste conceito com a epopeia marítima e com as incertezas associadas à navegação, designando então, a possibilidade de um perigo natural, mas excluindo a falha e responsabilidade humana.

No entanto, segundo este autor com o advento do Iluminismo e da industrialização, a conotação do termo risco torna-se mais extensiva e o seu significado passa a abarcar a probabilidade de um perigo natural ou originado pelo ser humano. (SOUSA 2013)

$\mathrm{Na}$ actualidade, de acordo com ZINN (2008), apud SOUSA (2013), o termo risco está associado ao perigo, referindo-se a algo que pode originar danos.

Por outro lado, o conceito é usado para expressar a probabilidade e extensão de um evento. Por fim, o conceito de risco pode adotar a conotação de assunção do risco, entendida pelo autor como uma avaliação subjetiva de ganhos e perdas. (SOUSA, 2013)

A palavra risco apresentou significados diferenciados ao longo do tempo, modificando-se até tomar a noção actual. De acordo com REBELO (I999), citado por DAGNINO e JUNIOR, (2007), no senso comum, o risco está vinculado à possibilidade de um dano, de insucesso ou de um acontecimento eventual que não depende exclusivamente da vontade dos agentes. 
Para MENDONÇA e LEITÃO, (2008), a noção de risco está ligada tanto às ciências da natureza quanto às ciências da sociedade e, respeitando-se esta dualidade e esta interface, entende-se o risco como um produto combinado: aquele de uma eventualidade e de uma vulnerabilidade.

De acordo com LOURENÇO e AMARO (2018), em função da causa que origina cada um deles, os riscos dividem-se em três grandes conjuntos, nomeadamente os riscos naturais (quando a sua causa tem origem em fenómenos da natureza), riscos antrópicos (quando resultam de acção humana) e riscos mistos (quando a sua causa tanto pode ser de origem natural como devida a causa humana).

Para IBGE (2005) risco é o produto de perigo e vulnerabilidade. KRON e THUMERER (2002) e HOGAN e MANDAROLA (2007), citados por OLIVEIRA, (20II) definem o risco como factor resultante do produto entre ameaça, exposição e vulnerabilidade.

Face as diferentes perceções acerca da definição do risco sob olhar de vários autores acima mencionados, e por razão desta diversidade, o termo risco recebe, um adjectivo que indica a origem do fenômeno perigoso, como natural, antrópico ou misto. Todavia, independente da origem, o risco sempre deverá recair, principalmente sobre o ser humano, pois a noção do risco apenas existe se alguém pode perder alguma coisa.

\subsection{Em busca do significado da vulnerabilidade}

De acordo com DUBOIS-MAURY e CHALINE (2003:2I), apud MENDONÇA e LEITÃO (2008), a vulnerabilidade “... aos riscos, quer sejam endógenos ou exógenos, é uma noção complexa, pois pode ter conteúdos humanos, econômicos, patrimoniais, tecnológicos e organizacionais que se diversificam no espaço e no tempo", ao que acrescentamos a cultura e o meio ambiente como conteúdos importantes.

No quadro de uma diversidade de linhas, a sistematização feita por CUTTER (1996), citado por CONY (2013), estabelece distinções que incluem uma perspectiva dinâmica e ampla. Para a autora, há três temas expressivos nos estudos da vulnerabilidade: I) a vulnerabilidade como uma condição pré-existente; 2) a 
vulnerabilidade como uma resposta matizada; e 3) a vulnerabilidade como perigos do lugar.

De acordo com CUTTER, (1996:532-533), apud CONY, (2013) a vulnerabilidade como uma resposta matizada, destaca as respostas e formas de lidar com os perigos, incluindo-se a resistência e a resiliência.

Considerando os eventos perigosos como construções sociais, para CUTTER, (1996:532) apud CONY, (2013), a vulnerabilidade matizada examina distúrbios crônicos, tais como as secas, a fome, mudanças climáticas ou mudanças ambientais. Para a autora, essa visão valoriza a construção social da vulnerabilidade, uma condição com origem em processos históricos e socioeconômicos que alteram a capacidade de indivíduos ou da sociedade para lidar com desastres e responder adequadamente a eles.

Ao lado da vulnerabilidade como uma condição pré-existente e da vulnerabilidade como uma resposta matizada, há uma terceira vertente, chamada por Cutter de vulnerabilidade como perigos do lugar. Essa visão combina as duas anteriores e acrescenta uma visão geográfica. Assim, a vulnerabilidade estaria associada tanto a riscos biofísicos, como a respostas sociais, mas em um domínio de área ou domínio geográfico específico (CUTTER, 1996:533). A autora propõe um refinamento da perspectiva que originou essa proposta, apresentando um modelo de vulnerabilidade que relaciona risco, mitigação, potencial de perigo, tecido social, vulnerabilidade social, contexto geográfico e vulnerabilidade biofísica e tecnológica. A interseção e interação tanto da vulnerabilidade social como da vulnerabilidade biofísica e tecnológica é que criariam a vulnerabilidade de lugares, em um processo geral interativo (CUTTER, 1996:535) apud (CONY, 2013).

Segundo CUNHA (2002), a combinação entre o factor Aléas ou Hazard e o factor Vulnerabilidade, talvez se traduza melhor como conceito de risco natural, se estes forem relacionados através de um operador de multiplicação, do que simplesmente através do operador função. Atendendo às considerações atrás descritas, a combinação destes conceitos cria a seguinte definição agregada: Risco (risk) = Perigosidade (hazard ou aléas) $x$ Vulnerabilidade. 
Deste ponto de vista, o risco é encarado como a perda potencial dos elementos ou sistemas expostos, resultante da junção da perigosidade com a vulnerabilidade (CARDONA, 2004), apud (SOUSA, 2013).

Nas ciências sociais, a vulnerabilidade decorre de fenômenos diversos, que afectam de forma diferenciada as pessoas e os grupos sociais. Factores como nível de renda, escolaridade, idade, gênero, acesso aos serviços públicos, habitação e participação política podem aumentar a predisposição à ocorrência de danos de diversas ordens, incluindo a própria morte, bem como expressam a capacidade de lidar com as crises e de aproveitar as oportunidades para melhorar sua situação de bem-estar (OLIMPIO e ZANELLA, 2008).

A vulnerabilidade está relacionada à capacidade da comunidade suportar e responder adequadamente a determinado evento. $O$ grau de vulnerabilidade da área geográfica e/ou da comunidade afectada é um dos factores preponderantes para a intensificação de suas consequências. (SOBRAL et al, 2010)

Deste modo, o risco corresponde a possibilidade de exposição a algum perigo e a vulnerabilidade corresponde à possibilidade de vivenciar experiências negativas em consequência da acção de agentes perigosos (internos ou externos e naturais ou artificiais), e reflecte a capacidade individual ou de uma sociedade em antecipar, preparar, responder e recuperar do desastre. A vulnerabilidade mantém com o risco uma relação de causalidade, pois o risco apenas existirá quando houver alguém ou alguma coisa que possa ser impactado.

\subsection{O risco de desastre natural}

O risco de desastre natural é a denominação preferida para fazer referência àqueles riscos que não podem ser facilmente atribuídos ou relacionáveis à acção humana. Embora, nos dias de hoje, essa seja uma tarefa cada vez mais difícil, REBELO (2003:II-22), apud DAGNINO e JUNIOR, (2007), apresenta a seguinte tipologia de riscos naturais: riscos tectônicos e magmáticos; riscos climáticos; riscos geomorfológicos, os mais típicos, tais quais ravinamento, de movimentações de massa, como desabamento ou deslizamento e outros riscos geomorfológicos como os 
decorrentes da erosão eólica e do descongelamento de neves de altitude e os riscos hidrológicos.

Para SOBRAL et all (2010), as ameaças entendidas como inundação, enchente, deslizamento de terra, etc. podem contribuir para a ocorrência e a severidade dos desastres naturais, mas a vulnerabilidade humana, resultante da pobreza e da desigualdade social, potencializa os riscos.

Desastre pode ser definido como "resultado de eventos adversos, naturais ou provocados pelo Homem, sobre um ecossistema vulnerável, causando danos humanos, materiais e ambientais e consequentes prejuízos econômicos e sociais”. (SOBRAL et al, 2010)

\section{UM OLHAR SOBRE A COMUNICAÇÃO NA CONSTRUÇÃO SOCIAL}

\section{I. Importância dos meios de comunicação social}

Segundo ESTEVES (2003:169), apud NUNES (2007), “a importância dos meios de comunicação nos nossos dias é um facto absolutamente iniludível e o

reconhecimento dessa importância apresenta-se tanto mais facilitado quanto as sociedades humanas atingem níveis mais elevados de desenvolvimento".

Para este autor, na nossa sociedade a comunicação social assume uma certa importância já que suporta conteúdos que contribuem para os processos de produção e construção, de reprodução e reconstrução e de representação social da realidade e da cultura, contribuindo, também, na determinação da história das civilizações, das sociedades e das culturas.

\subsection{A comunicação do risco de desastres naturais}

Comunicação de riscos é o processo de comunicar sobre riscos à saúde, ao meio ambiente natural e urbano e à segurança da população (CUNHA, R., 2008).

De acordo com MONTEIRO (2009:160), a comunicação de riscos pode ser definida como "um processo interativo de troca de informações e de opiniões entre indivíduos, grupos e instituições, envolvendo mensagens sobre a natureza do risco e 
outras, que expressam preocupações, opiniões ou reações às mensagens de risco ou os arranjos legais e institucionais para o gerenciamento dos riscos.

Refere-se "a qualquer comunicação pública ou privada para trocar informações e opiniões com os indivíduos sobre a existência, a natureza, a forma, a gravidade ou a aceitabilidade dos riscos" .... "Focaliza uma transferência intencional de informações de especialistas para não especialistas, com vista a responder às preocupações ou necessidades do público leigo quanto a um determinado perigo - real ou percebido". (MONTEIRO, 2009:I60)

Deste modo, de acordo com NETO e AREOSA (2019), apud (CUNHA e CUNHA, 2002), pode assumir-se que a comunicação do risco implica o envolvimento de diferentes públicos e partes interessadas, que vão trocar dados e informações entre si relativamente à natureza e características dos riscos e das exposições que existem ou podem acontecer.

A comunicação de riscos é um recurso para divulgar as informações sobre o risco ao público em geral, fornecendo conteúdo suficiente para que as pessoas compreendam os aspectos técnicos dos riscos a que estão expostas e possam decidir sobre esses riscos.

Denote-se que a gestão do risco diz respeito ao conjunto de actividades desenvolvidas para identificar os perigos e avaliar os riscos existentes num determinado contexto com o intuito de serem idealizadas e implementadas medidas que permitam controlar os riscos sinalizados (NETO, 2014).

A comunicação do risco é uma das componentes do processo de gestão de desastres naturais, funcionando também como elemento de ligação das diferenças etapas da gestão do risco de desastres naturais em busca das melhores formas de se lidar com o desastre.

A comunicação do risco, enquanto processo interativo de troca de informações e opiniões sobre o risco, é algo necessário desde o enquadramento da situação de risco até à implementação e acompanhamento das medidas de gestão. É um meio para assegurar o intercâmbio de informações entre os profissionais do risco e de comunicar adequadamente o risco ao mundo exterior (RENN, 2005), apud (SOUSA 2013). 
Desse modo, de acordo com FURTADO, (2014), buscando o aperfeiçoamento na forma de se comunicar, a instituição conquistará a credibilidade da mídia e dos demais públicos, por se preocupar em repassar informações precisas sobre o cenário de desastre. Se manifestar e prestar esclarecimentos demonstra seriedade e respeito com os envolvidos, bem como controle da situação e segurança. O responsável pela comunicação deve definir as acções de comunicação em um período de crise de forma prévia, garantindo uma actuação eficaz durante o desastre.

No processo da comunicação do risco de desastre natural a participação das Mídias é fundamental, e o texto Comunicação de riscos e de desastres (BRASIL, 2010), explica:

Podemos dizer que, na condição de desastre, a imprensa precisa: Divulgar as
informações de forma responsável, priorizando sempre o bem-estar e a
segurança da população; Respeitar as determinações das equipes que actuam
no gerenciamento do desastre e contribuir com elas; Corrigir rumores falsos
sobre a situação; Apurar e levar informações até a população que ajudem a
minimizar os danos e os prejuízos; Repassar orientações corretas e de
interesse público com agilidade; Consultar fontes que enriqueçam e
acrescentem outros
dados às informações disponíveis; Avaliar as informações antes da
divulgação e Agir de forma responsável e comprometida com a redução dos
riscos. Se, desde o início do desastre, a instituição efectuar a comunicação de
forma permanente, ela demonstrará que está enfrentando o problema de
maneira responsável. (BRASIL, 2oIo)

De acordo com (MCCOMMAS, 2006; RENN, 2005), apud SOUSA (2013), como resultado desta evolução, a comunicação do risco é hoje entendida como um processo multidisciplinar e multidimensional de interação entre indivíduos, grupos e instituições que expressam preocupações, opiniões e reações sobre as causas, características e consequências de um risco. Nesse sentido, o risco é hoje encarado como um constructo social, cultural e psicologicamente alicerçado

\subsection{Metodologias de comunicação do risco de desastres naturais}

Utilizando a terminologia de HOPPNER et al. (2010), apud SOUSA (2013), o modo de comunicação pode ser unidirecional, quando a transferência de informação entre os comunicadores se dá apenas num sentido, ou bidirecional, se a informação flui em ambas as direcções entre os actores/ intervenientes, podendo assumir a forma 
escrita (jornais, cartas, relatórios, panfletos, etc.), verbal (conversas, apresentações, debates, etc.) ou não-verbal/ visual (filmes, gráficos, linguagem gestual, expressões faciais, entre outras). Os canais de comunicação ou são directos (face-to-face) ou indirectos (mediados) e podem visar públicos específicos, constituídos por um reduzido número de indivíduos, ou procurar atingir audiências mais difusas, compostas por um número mais elevado de atores. Considerando a dimensão temporal, a comunicação pode ser pontual, periódica ou uma atividade contínua, consoante a sua duração. (HOPPNER et al. 2010), apud (SOUSA 2013)

Segundo SOBRAL, et al (2010), os Sistemas de Informação Geográfica (SIG) são citados como importantes ferramentas e instrumentos metodológicos fundamentais para a gestão de desastres naturais, através da realização de análises espaciais complexas. Podem subsidiar a construção de um Atlas de risco de desastres em nível regional. Possuem bancos de dados que permitem a integração de diferentes dados e informações de naturezas diversas (demográficas, sociais, ambientais, sanitárias, geológicas etc.) para a avaliação rápida das necessidades da população afectada. (SOBRAL, et al 2010).

\section{MATERIAIS E MÉTODOS}

Para o alcance dos objectivos traçados aplicou-se à abordagem qualitativa, que de acordo com OLIVEIRA (20II: 25), compreende actividades ou investigação que podem ser denominadas específicas, tendo sido aplicadas as pesquisas documental e bibliográfica por meio de consulta a diversos documentos e obras que abordam os assuntos tratados, tendo sido feita análise e selecção do essencial para a produção deste trabalho.

\section{RESULTADOS}

\section{I O risco de desastres naturais em Moçambique}

Moçambique é um país que enfrenta várias ameaças aos desastres naturais. Devido à sua morfologia e condições geográficas, o país está exposto a eventos 
extremos, sendo os mais frequentes as cheias, ciclones e secas. A vulnerabilidade e risco de desastres naturais resulta, principalmente, da sua localização na foz de rios internacionais, a existência de zonas áridas e semiáridas; a longa extensão do território nacional localizado na zona de convergência intertropical, a extensa zona costeira que sofre a influência de ciclones tropicais.

O mapa da localização geográfica de Moçambique ilustra a disposição do relevo e as bacias hidrográficas, algumas das quais partilhadas com alguns países vizinhos a montante, sendo que Moçambique localiza-se a jusante.

\section{Figura 1. Mapa de Localização geográfica de Moçambique}

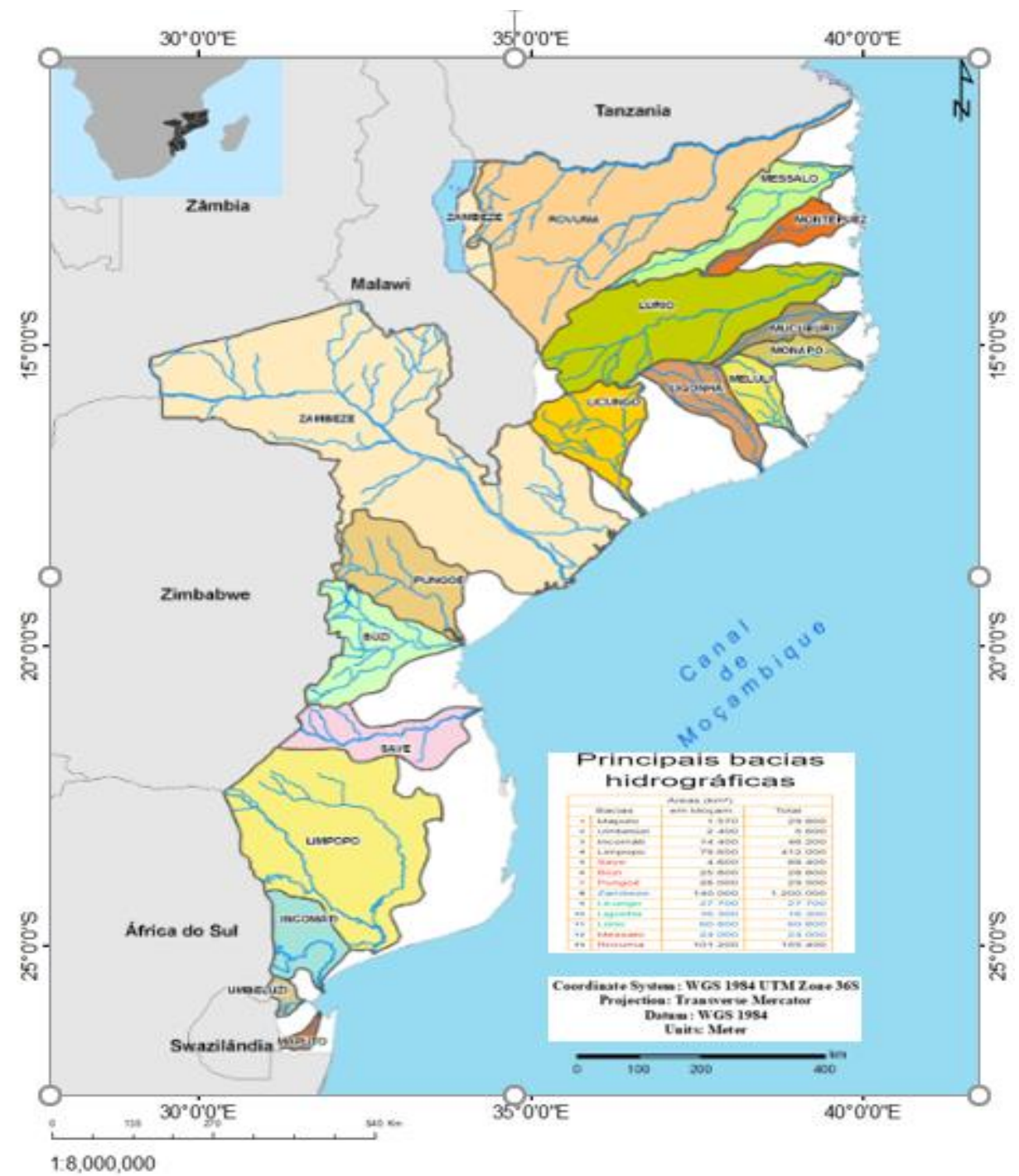

Fonte: Autores. Laboratório de SIG Universidade Pedagógica de Maputo. (202I) 
Nos últimos anos, a elevada frequência, alternância e intensidade dos desastres naturais, agravada pelas mudanças climáticas, passaram a constituir uma grande ameaça aos esforços de desenvolvimento nacional, visto que funcionam como um entrave à aceleração do crescimento económico e tendem a corroer os ganhos já alcançados.

Os ciclones, cheias e secas constituem as principais ameaças e maior preocupação para o País, e sua frequência, magnitude e severidade poderão aumentar devido às mudanças climáticas, sendo que 58 por cento da população está exposta a pelo menos duas ameaças naturais, sobretudo secas, cheias e ciclones.

Os desastres afectam negativamente a renda e produtividade dos agregados familiares rurais, devido a sua dependência pela agricultura de sequeiro e da exploração dos recursos naturais. Anualmente, as secas custam ao sector agrícola cerca de 20 milhões de dólares americanos.

A economia moçambicana é vulnerável aos desastres naturais, devido à sua altamente dependência pelo desempenho da agricultura. As cheias e ciclones constituem uma ameaça à segurança das infraestruturas e da comunidade escolar. $\mathrm{O}$ sector de educação é o sector social mais afectado pelos desastres no País. Pelo menos 34.742 salas de aula estão em risco de serem afectadas por pelo menos uma ameaça natural por ano. (CONSELHO DE MINISTROS, 2013, 2018).

\subsection{Quadro institucional da gestão e redução do risco de desastres em Moçambique.}

O Instituto Nacional de Gestão do Risco de Desastres (INGD) é a instituição do Estado responsável pela coordenação da gestão do risco de desastres em Moçambique, uma entidade do Estado criada para liderar a coordenação da prevenção e mitigação dos desastres no País que funciona em colaboração com o Instituto Nacional de Meteorologia.

\subsection{Comunicação do risco de desastres naturais em Moçambique}

Moçambique tem um sistema de gestão de risco de desastres com reconhecido mérito de funcionamento em vários níveis, que serve como ponto de partida da estratégia para a redução do risco de desastres. 
Neste contexto, afigura-se pertinente a adopção de uma estratégia e o respectivo plano de acção que delineia as linhas orientadoras, acções e recursos necessários para proteger as infra-estruturas vitais para o desenvolvimento socioeconómico do País e capacitar os cidadãos e comunidades a reduzirem o seu nível de vulnerabilidade aos efeitos calamitosos de maior magnitude suscitados pela influência das mudanças climáticas e uma das várias estratégias e produzir e difundir conhecimentos com um padrão internacional sobre mudanças climáticas e estratégias de adaptação para redução do risco de desastres (INGC, 2012).

A Lei n. ㄷ/2014 de 20 de Junho, no seu artigo 2I (Informação), sugere:

I- Os órgãos de comunicação social, públicos ou privados, devem providenciar informação adequada sobre a gestão das calamidades/desastres;

2. Declarado um alerta, os órgãos de comunicação social difundem, a nível nacional, ou local, os comunicados sobre a gestão das calamidades.

As comunidades locais participam activamente na gestão do risco de desastres através dos Comités Locais de Gestão do Risco de Calamidades (CLGRC). A existência e pleno funcionamento dos comités em todas as áreas de maior risco constitui o maior desafio para o País reforçar a capacidade local de prontidão e resposta à emergência, mediante utilização adequada da informação de aviso prévio. $\mathrm{O}$ acesso à informação e disseminação dos avisos prévios para o reforço da prontidão e resposta as cheias e ciclones ainda apresenta deficiências e sua cobertura é desconhecida.

O Governo está a implementar desde 2005, um sistema de aviso prévio para cheias e ciclones, baseado numa escala de cores. Este sistema serve de base para a emissão e disseminação dos correspondentes alertas pelo INGD, às populações em risco, depois de recebidos os respectivos avisos de cheias e ciclones emitidos pela Direcção Nacional de Gestão dos Recursos Hídricos (DNGRH) e pelo Instituto Nacional de Meteorologia (INAM).

Os alertas são disseminados pelo Centro Nacional Operativo de Emergência (CENOE) /INGD em colaboração com estruturas locais (ex: governo provincial e distrital) que, por sua vez, compartilham com os rádios comunitárias e Comités 
Locais de Gestão de Riscos para uma divulgação mais ampla para a acção ou reacção comunitária adequada ou proporcional ao evento.

O sector de telecomunicações de emergência assegura o estabelecimento de serviços de comunicação de Voz via Rádio e transmissão de dados. Este serviço tem como finalidade providenciar um meio de comunicação de emergência seguro e confiável.

De acordo com a Lei número Io/2020 de 24 de Agosto, (Lei de Gestão do risco de desastres), o artigo 4 (princípio da informação), estabelece que todos os intervenientes na gestão e redução do risco de desastres devem manter informadas as instituições e pessoas sobre o processo de gestão e redução do risco de desastres;

- O Princípio da educação e sensibilização públicas consagra o melhoramento da transmissão ou difusão de valores e práticas orientado para a gestão e redução do risco de desastres a todos os níveis;

$\mathrm{O}$ artigo ${ }_{14}$ (Subsistema de aviso prévio e de alerta), orienta:

I. O subsistema de aviso prévio e de alerta visa monitorar as ameaças, prever, avaliar o risco de desastres e disseminar, atempadamente, informação para a tomada de medidas preventivas pelas comunidades potencialmente em risco e pelas entidades competentes;

2. O subsistema de aviso prévio e de alerta compreende, designadamente: a) fase de aviso prévio; b) fase de alerta.

Artigo I5 (Fase de aviso prévio) I. A fase do aviso prévio inclui as seguintes componentes: a) vigilância e previsão; b) conhecimento do risco; c) serviço de monitoria; d) disseminação e comunicação; e) capacidade de resposta aos alertas; f) preparação para a resposta.

Artigo i6 (Fase de Alerta) i. A fase do alerta compreende:

O alerta amarelo, que é activado quando há previsão de ocorrência de um fenómeno susceptível de causar danos humanos, ambientais e materiais; o alerta laranja, que é activado quando há iminência de ocorrência de um fenómeno capaz de causar danos humanos, ambientais e materiais, mas com a possibilidade de reversão; o alerta vermelho, que é activado quando o fenómeno é irreversível e prevê-se a 
ocorrência de danos humanos, ambientais e materiais que possam se transformar em desastre de grande magnitude.

\section{Artigo 25 (Informação sobre o risco de desastres) orienta:}

I. A informação sobre o risco de desastres inclui estudos, informações e mapeamentos necessários para entender os seus vectores e os factores subjacentes.

\subsection{A Importância da comunicação do risco de desastres naturais em Moçambique}

Moçambique é classificado como um dos países mais vulneráveis ao risco de desastres naturais. A vulnerabilidade e risco de desastres naturais resulta da sua localização na foz de rios internacionais, a existência de zonas áridas e semiáridas; a longa extensão do território nacional localizado na zona de convergência intertropical, a extensa zona costeira que sofre a influência de ciclones tropicais e a existência de zonas sísmicas activas. Deste modo é importante que o país adopte por um sistema de comunicação de risco de desastres naturais mais robusto e eficiente, pois, a comunicação de risco revela-se de extrema importância na gestão do risco do desastre, e de acordo com JESUS, (2013:14), como uma componente do processo de gestão do risco que, por sua vez, consiste na definição de opções no âmbito do controlo do risco. Deste modo, este é o processo que fornece a informação sobre a qual o Governo e os próprios indivíduos baseiam a sua tomada de decisão.

A comunicação do risco providencia informação acerca da probabilidade de exposição a um determinado factor e acerca da natureza e extensão das suas consequências. Além disso, sempre que as consequências resultantes da exposição a um determinado factor sejam consideradas desconhecidas ou incertas, a comunicação do risco transmite informação suficiente no que concerne à origem e extensão do grau de incerteza.

A comunicação do risco assume um papel preponderante, na medida em que não só auxilia a compreender a percepção da população e a antecipar a resposta da comunidade perante as acções concretizadas pelas Entidades, mas também 
potencializa a eficácia das decisões no âmbito das decisões da gestão do risco através do envolvimento da população (DGS, 2007), apud (JESUS, 2013:14).

Nesse sentido, para ALMEIDA e BERTUCCI (1993), a Comunicação de Risco configura-se como uma estratégia de inovação para as práticas de Sustentabilidade, tanto econômica, social e ambiental, já que proporciona a articulação com vista a diminuir as incertezas em relação aos possíveis riscos e auxiliar os indivíduos nas suas escolhas e projecções de diferentes futuros, o que garante melhor desempenho ambiental e social.

A comunicação de risco é, portanto, parte integrante do processo de gerenciamento de risco, responsável por integrar os possíveis afectados e informá-los sobre que maneiras e procedimentos adotar perante ameaças e inseguranças, causadas por riscos (ALMEIDA e BERTUCCI, 1993).

De acordo com TORQUATO (2008:123), em situação de emergência e/ou desastres, a comunicação é importante pelo facto de ter as seguintes funções:

- A comunicação como base de cidadania (direito à informação): nesse caso, a comunicação deve ser entendida como um dever da administração pública $e$ um direito dos usuários dos serviços;

- A comunicação como forma de orientação aos cidadãos (função educativa): nesse aspecto, a comunicação assume papel de fonte de educação, pela transmissão de valores, ideias e cargas informativas que sedimentarão a bagagem de conhecimento dos receptores;

- E, por fim, a comunicação como instrumento a serviço da verdade (função ética). A verdade deve ser a fonte de inspiração da comunicação pública. (TORQUATO 2008:123)

De acordo com SOUSA, (2013), a comunicação do risco permite, que os diferentes interlocutores e a sociedade de um modo geral entendam os riscos a que se encontram expostos e reconheçam o seu papel no processo de governança do risco.

Por outro lado, o carácter deliberadamente bidirecional da comunicação, possibilita a participação dos diferentes intervenientes, quer no processo de análise e compreensão, quer no processo de tomada de decisão, implementação e regulação das intervenções. (SOUSA, 2013) 
Uma vez tomadas as decisões, no âmbito da gestão do risco, a comunicação permite explicar as razões dessas decisões e informar o público sobre as oportunidades e desafios de mitigação e minimização das suas consequências, incluindo as responsabilidades inerentes a cada um dos interlocutores. (ibid)

Para JESUS (2013:14), a comunicação do risco é considerada um componente do processo de gestão do risco que, por sua vez, consiste na definição de opções no âmbito do controlo do risco. Deste modo, este é o processo que fornece a informação sobre a qual o governo e os próprios indivíduos baseiam a sua tomada de decisão.

A comunicação do risco inclui todas as mensagens que são consideradas enquanto suporte da tomada de decisões no processo de gestão do risco. Por tanto, a comunicação do risco abrange anúncios, avisos e instruções emitidas pelos especialistas e dirigidas ao público leigo. Visa, também a divulgação de informação sobre o risco; sobre as fontes de informação; sobre as crenças pessoais e sentimentos referentes aos riscos e aos consequentes danos (JESUS, 2013).

Respeitando as diferentes funções da comunicação mencionadas, percebemos a importância da comunicação na esfera pública moçambicana e na gestão do risco de desastres naturais, num país vulnerável a ocorrência de desastres naturais. Além disso, ainda podemos perceber a importância da comunicação com o nosso público, de forma mais comprometida com a divulgação das açcões acerca dos riscos de desastres naturais e deste modo a reduzir os impactos dos desastres naturais sobre o ambiente e a sociedade, num país que enfrenta grandes desafios de combate à pobreza.

\section{CONCLUSÃO}

Diante dos riscos e vulnerabilidade de que a população moçambicana se encontra exposta aos desastres naturais, torna-se importante a adopção e aplicação de mecanismos para a minimização dos riscos e vulnerabilidades com vista a redução dos impactos negativos derivados da ocorrência dos desastres naturais. Nesse sentido, apoiar a população, instituições e comunidades a antecipar, resistir, enfrentar e recuperar de desastres naturais, é uma tarefa exigente e complexa, mas simultaneamente um objectivo que se deve concretizar aplicando várias ferramentas 
de gestão do risco. Neste contexto, a comunicação do risco de desastres naturais surge cada vez mais referenciada como uma ferramenta de gestão do risco e um processo extremamente importante na difusão de conhecimentos, na modificação e reforço de condutas, valores e doutrinas sociais, capazes de contribuir para a prevenção e minimização do risco e para o desenvolvimento de sociedades mais resilientes face aos desastres naturais. Porém a diversidade de factores e intervenientes envolvidos nos processos de comunicação em torno do risco e do desastre, gera situações de interação que exigem estratégias e modelos capazes de responder à complexidade e natureza do risco, mas simultaneamente corresponder às expectativas de bem-estar e segurança das sociedades actuais. Uma vez tomadas as decisões, no âmbito da gestão do risco, a comunicação permite explicar as razões dessas decisões e informar o público sobre as oportunidades e desafios de mitigação e minimização das suas consequências, incluindo as responsabilidades inerentes a cada um dos intervenientes. Nesse sentido, a comunicação é também a chave para a criação de confiança nas estruturas de gestão do risco de desastres naturais. Em Moçambique, o Instituto Nacional de Gestão do Risco de Desastres (INGD) é a instituição do Estado responsável pela coordenação da gestão do risco de desastres em Moçambique, uma entidade do Estado criada para liderar a coordenação da prevenção e mitigação dos desastres no País

\section{REFERÊNCIAS BILIOGRÁFICAS}

ALMEIDA, Ana Luísa de Castro e BERTUCCI, Janete Lara de Oliveira. Gestão estratégica de stakeholders: Aspectos relevantes na definição de políticas de relacionamento. In: MARCHIORI, M. Comunicação e organização: reflexões, processos e prática, p. I9I207. 1993.

BRASIL. Programa 2040: gestão de riscos e resposta a desastres. Ministério da Integração do Brasil. 2013.

CARVAlHO, C. P.; GOMES, R. S., O Rito do Processo de Comunicação de Crise como indicador de Sustentabilidade Organizacional. In. Revista de Estudos da Comunicação. Curitiba, v. Io, n. 23, p. 267-274. 2009 
CONY, Lúcia. Urbanização, ambiente, risco e vulnerabilidade: Em busca de uma construção interdisciplinar. Cadernos Metrópole. Vol. 15.n 29. São Paulo. Pontifícia Universidade Católica de São Paulo. 2013.

CUNHA, R. D. S. Avaliação da estratégia da comunicação de riscos ambientais na preparação do público para acidentes de grande porte: estudo de caso do Plano APELL em São Sebastião. São Paulo. Dissertação (Mestrado em Comunicação, Educação e Administração). Universidade São Marcos. 2008.

CUNHA, Ícaro e CUNHA, Raquel. A comunicação do risco na preparação para emergências ambientais. São Paulo. Climatologia e estudos da paisagem. Vol. 7.n.I.2. 2002.

DAGNINO, Ricardo e JÚNIOR, Salvador. Risco ambiental: conceitos e aplicações. Climatologia e Estudos da Paisagem Rio Claro - Vol.2 - n.2 - 2007.

FURTADO, Januário (Org). Gestão de desastres e acções de recuperação. Florianópolis. Universidade Federal de Santa Catarina. 2014.

IBGE. A Vulnerabilidade a Desastres Naturais. Perfil dos municípios brasileiros. Rio de Janeiro. 2005.

INGC (Instituto Nacional de Gestão de Calamidades). Respondendo as mudanças climáticas em Moçambique. Maputo. 2012.

JESUS, Sandra. Comunicação do risco natural em Portugal. (Dissertação de Mestrado). Lisboa. Instituto Politécnico de Lisboa. 2013.

LOURENÇO, Luciano e AMARO, António (Coord). Riscos e crises. Da teoria a plena manifestação. Coimbra. Imprensa da Universidade de Coimbra. 2018.

MENDONÇA, Francisco e LEITÃO, Sanderson. Riscos e vulnerabilidade socio ambiental urbana: Uma perspectiva a partir dos recursos hídricos. Paraná. Universidade Federal do Paraná. 2008. 
MONTEIRO, Mário. Ciência e Risco: as controvérsias como procedimento da comunicação pública em um contexto democrático. Tese de Doutoramento. Brasília Universidade de Brasília. 2009.

NETO, H. V. Sebenta de Técnicas de Informação. Comunicação e Negociação. Gaia. ISLA - Instituto Politécnico de Gestão e Tecnologia. 2014.

NUNES, Sónia. Sociedade de informação. Coimbra. Universidade de Coimbra. 2007.

OLIMPIO, João e ZANELLA, Maria. Riscos naturais: Conceitos, componentes e relações entre natureza e sociedade. Ceara. Instituto Federal de Educação. 2017.

OLIVEIRA, Alzira Marques. Indicadores de vulnerabilidade e risco socio ambiental para prevenção e mitigação de desastres naturais na bacia do rio Jari. Dissertação de Mestrado. Macapá. Universidade Federal do Amapá. 20 Ir.

OLIVEIRA, M. Metodologia Científica: Um manual para a realização de pesquisa em administração. Catalão. Universidade Federal de Goiás. 201 .

PRANDEL, Jorge. (Org). Redução de desastres. Métodos e práticas. Ponta Grossa. Atena Editora. 2019.

QUADRO DE ACÇÃO DE SENDAI PARA A REDUÇÃO DE RISCOS DE DESASTRES 2015-2030.

REBELO, Fernando. Teoria do risco numa perspectiva geográfica. Cadernos de Geografia, n. I8. Coimbra. 1999.

REPÚBLICA DE MOÇAMBIQUE (Conselho de Ministros). Plano de Contingência para a época chuvosa e de ciclones 2013-2014. Maputo. 2013.

REPÚBLICA DE MOÇAMBIQUE. Lei n.ํำ $15 / 2014$ de 20 de Junho: Lei de Gestão de calamidades. Maputo. 2020.

REPÚBLICA DE MOÇAMBIQUE. Lei n.ํำ ıo/2020: Lei de Gestão e Redução do Risco de Desastres. Maputo. 2020.

SERRES, Manuel. O contracto natural. Lisboa. Instituto Piaget. 2000. 
SOBRAL, André et al. Desastres naturais - Sistema de informação e vigilância: Uma revisão da literatura. Rio de Janeiro. Fundação Oswaldo Cruz. 20 ı.

SOUSA, José. A comunicação do risco na minimização de desastres naturais na região autónoma da Madeira. (Dissertação de Mestrado em Gestão do Território). Lisboa. Universidade Nova de Lisboa. 2013.

TORQUATO, Gaudêncio. Tratado da comunicação organizacional e política. São Paulo. Cengage Learning. 2008. 